\title{
Decomposing Cavities in Digital Volumes into Products of Cycles ${ }^{\star}$
}

\author{
Ainhoa Berciano ${ }^{1}$, Helena Molina-Abril ${ }^{2}$, Ana Pacheco ${ }^{2}$, \\ Paweł Pilarczyk ${ }^{3}$, and Pedro Real ${ }^{2}$ \\ 1 Departamento de Didactica de la Matematica y de las CC. Experimentales, \\ Universidad del Pais Vasco-Euskal Herriko Unibertsitatea, \\ Ramon y Cajal, 72. 48014-Bilbao (Bizkaia), Spain

ainhoa.berciano@ehu.es \\ 2 Departamento de Matematica Aplicada I, Universidad de Sevilla, \\ Avenida Reina Mercedes, Spain \\ \{habril, ampm, real\}@us.es \\ http://ma1.eii.us.es/ \\ 3 Centro de Matemática, Universidade do Minho, \\ Campus de Gualtar, 4710-057 Braga, Portugal
}

http://www.pawelpilarczyk.com/

\begin{abstract}
The homology of binary 3-dimensional digital images (digital volumes) provides concise algebraic description of their topology in terms of connected components, tunnels and cavities. Homology generators corresponding to these features are represented by nontrivial 0 cycles, 1-cycles and 2-cycles, respectively. In the framework of cubical representation of digital volumes with the topology that corresponds to the 26-connectivity between voxels, we introduce a method for algorithmic computation of a coproduct operation that can be used to decompose $2-$ cycles into products of 1 -cycles (possibly trivial). This coproduct provides means of classifying different kinds of cavities; in particular, it allows to distinguish certain homotopically non-equivalent spaces that have isomorphic homology. We define this coproduct at the level of a cubical complex built directly upon voxels of the digital image, and we construct it by means of the classical Alexander-Whitney map on a simplicial subdivision of faces of the voxels.
\end{abstract}

Keywords: homology, cubical homology, cubical set, cell complex, digital image, cavity, cycle, Alexander Whitney diagonal, chain homotopy, algebraic gradient vector field.

* This work has been partially supported by "Computational Topology and Applied Mathematics" PAICYT research project FQM-296, "Andalusian research project" PO6-TIC-02268, Spanish MEC project MTM2006-03722 and the Austrian Science Fund under grant P20134-N13. 


\section{Introduction}

Over the recent decades, considerable progress has been made in the development of topological methods for the analysis of digital images. In particular, effective algorithms and efficient software for the computation of homology groups and their generators have been under heavy development; see [1|2|34 $516|7| 8|9| 10$. for some of this work. These tools have already proved their usefulness in applications, e.g. 11/2 131415], and their potential in multi-dimensional digital image analysis is undeniable.

An $n$-dimensional binary digital image can be perceived as a union of closed $n$-dimensional hypercubes in $\mathbb{R}^{n}$ with respect to a uniform rectangular lattice with the topology induced from $\mathbb{R}^{n}$, which corresponds to the topology of $\left(3^{n}-1\right)$-connectivity between the $n$-dimensional pixels $(26$-connectivity in the 3 -dimensional case). The homology of such a set provides information on the number of connected components and holes of various dimensions. Computing homology generators allows to locate the connected components in the digital image and enclose the various holes geometrically.

A homology generator of dimension 1 is represented at chain level by a nontrivial 1-cycle and encloses some tunnel. A homology generator of dimension 2 is represented by a nontrivial $2-$ cycle and encloses some cavity. In this paper we are interested in decomposing 2-cycles into products of 1-cycles (which may turn out to be trivial in some cases), in order to classify the different types of cavities in digital volumes, and identify the related tunnels, if any (see Fig. 11). Such a method will provide a means of more thorough analysis of the geometry of the digital volumes, important e.g. for the purpose of structural pattern recognition. It will also allow to distinguish certain substantially different (homotopically non-equivalent) cases that give rise to isomorphic homology groups.

In order to address the problem in question, we consider the AlexanderWhitney diagonal, a canonical associative coproduct that is defined on the chain complex canonically associated to a simplicial complex $K$, and factorizes higherdimensional cycles into lower-dimensional ones. We adapt this theoretical machinery into the context of digital imagery. We work directly at the level of the cubical complexes inferred from the cubical voxels, their faces, edges and vertices. However, our approach is different from the classical Serre diagonal [16].

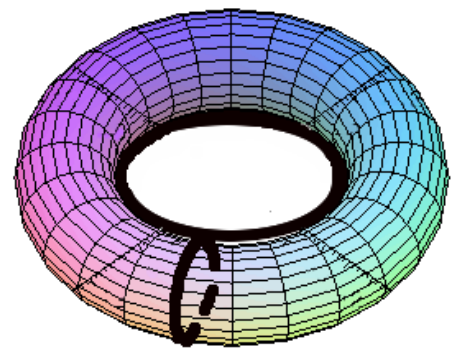

Fig. 1. Decomposing the cavity of a torus into cycles 
In fact, we do not use here cocycles or cohomology notions, but we remain at the easier level of cycles and homology.

Although the methods developed in this paper are dimension-independent and are valid in a very generic context, for the sake of clarity of presentation we restrict our attention to 3-dimensional binary digital images (also referred to as digital volumes), and we consider coefficients in the ring $\mathbb{F}_{2}$.

Our approach to the computation of the cubical version of the AlexanderWhitney diagonal for cavities in a digital volume can be summarized as follows. First, given a digital volume $K$, we represent it by means of a cubical chain complex, as described in Section 2. We compute its homology along with homology generators and a homology gradient vector field [7]. The latter object captures the deformation process for obtaining the minimal homological expression for $K$, and allows to instantly determine the homology class of every cycle. We describe this construction in Section 3. Then we specify a simplicial-valued diagonal for each cubical 2-cell using two basic techniques: simplicial subdivision of the cartesian product of simplices and chain homotopy equivalence. We express the simplicial result in terms of cubical 1-chains, and in this way we derive an explicit formula for the cubical version of this diagonal in Section 4. Finally, we apply the Alexander-Whitney diagonal to each representative nontrivial 2-cycle (homology generator) by means of the natural linear extension of the diagonal defined for the 2 -cells. The overall method combining all these mechanisms is described step by step in Section 5. Examples of the application of this algorithm are discussed in Section 6 .

\section{Simplicial and Cubical Complexes}

In this section we introduce cubical cell complexes which we use to represent binary digital images, and we also mention simplicial complexes which will be used as an intermediate step in the construction of the Alexander-Whitney diagonal for cubical complexes in Section 4.

Throughout the paper, we consider the finite field $F_{2}=\{0,1\}$ as the ground ring of coefficients, which simplifies many formulas and makes the entire construction reflect better the combinatorial aspect of this approach. Note that the homology of geometric objects embedded in $\mathbb{R}^{3}$ is torsion free, so we do not lose any information in this context by this choice of coefficients.

Let $K$ be an $n$-dimensional cell complex. Denote the set of its $q$-dimensional cells by $K^{(q)}$. The corresponding chain complex $\left(C_{q}(K), \partial_{q}\right)_{q \in \mathbb{Z}}$ consists of the fields of $q$-chains $C_{q}$ over $\mathbb{F}_{2}$, whose generators correspond to the cells in $K^{(q)}$, and a family of homomorphisms $\partial_{q}: C_{q} \rightarrow C_{q-1}$ such that $\partial_{q-1} \circ \partial_{q}=0$, which correspond to geometric boundaries of cells. Each $q$-chain $c \in C_{q}$ is then a formal sum $\sigma_{1}+\cdots+\sigma_{k}$ of selected cells $\sigma_{i} \in K^{(q)}$. We denote $\sigma \in c$ if $\sigma \in K^{(q)}$ is a summand of $c$. Note that $C_{q}(K)=0$ whenever $q<0$ or $q>n$.

The case of a simplicial complex is well established [17], and the boundary map $\partial_{q}$ on each $q$-simplex $\sigma=\left\langle v_{0}, \ldots, v_{q}\right\rangle$ is defined as follows: $\partial_{q}(\sigma)=$ $\sum\left\langle v_{0}, \ldots, \hat{v}_{i}, \ldots, v_{q}\right\rangle$, where the hat over $v_{i}$ means that $v_{i}$ is omitted (see Fig. 2 for an illustration of the boundary of a 2-simplex). 

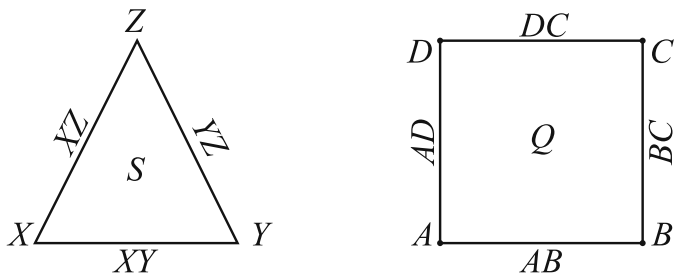

Fig. 2. The boundary of a 2-simplex $S$ (left) and a 2-cube $Q$ (right) over $\mathbb{F}_{2}: \partial_{2} S=$ $X Y+Y Z+X Z$ and $\partial_{2} Q=A B+B C+D C+A D$. The boundary of each edge consists of the two endpoints, e.g., $\partial_{1} A B=A+B$. It is easy to see that in both cases $\partial_{1} \circ \partial_{2}=0$.

The case of a cubical complex is less typical, so we recall some definitions in order to avoid any ambiguity. The reader is referred to [5] for a comprehensive introduction. An elementary interval is an interval of the form $I=[k, k+1]$ (the non-degenerate case) or a set $I=\{k\}$ (the degenerate case), where $k \in \mathbb{Z}$. An elementary cube in $\mathbb{R}^{n}$ is the cartesian product of $n$ elementary intervals, and the number of non-degenerate intervals in this product is its dimension.

In order to define the boundary of a $q$-cube $\sigma=I_{1} \times \cdots \times I_{n} \in \mathcal{K}_{q}^{n}$ (see Fig. 2 for an illustration of $\partial_{2}$ ), denote by $k_{1}, \ldots, k_{q}$ those indices that correspond to non-degenerate intervals $I_{k_{j}}=\left[a_{j}, b_{j}\right]$ in $\sigma$. Define $A_{k_{j}} \sigma:=I_{1} \times \cdots \times I_{j-1} \times$ $\left\{a_{j}\right\} \times I_{j+1} \times \cdots \times I_{n}$ and $B_{k_{j}} \sigma:=I_{1} \times \cdots \times I_{j-1} \times\left\{b_{j}\right\} \times I_{j+1} \times \cdots \times I_{n}$. Then $\partial_{q}(\sigma):=\sum_{i=1}^{q}\left(A_{k_{i}} \sigma+B_{k_{i}} \sigma\right)$.

We identify an $n$-dimensional binary digital image with the set $K^{(n)}$ of $n$ dimensional elementary cubes in $\mathbb{R}^{n}$ corresponding to its black pixels (in $2 \mathrm{D}$ ), or voxels (in $3 D$ ), or $n$-dimensional picture elements (in general). We add all the lower-dimensional cubes contained in the cubes in $K^{(n)}$ in order to obtain a cell complex $K$ that represents the digital image.

\section{Homology of Cell Complexes}

In this section we describe a homology computation procedure for finite cell complexes, in which a chain contraction is constructed in addition to the homology module and homology generators. As it will be made clear in Section 5 this construction is crucial for the successful method for the computation of the Alexander-Whitney diagonal.

Denote a cell complex under consideration by $(K, \partial)$, where $\partial: C(K) \rightarrow C(K)$ is its boundary map. A chain $a \in C_{q}(K)$ is called a $q-$ cycle if $\partial_{q}(a)=0$. If $a=\partial_{q+1}\left(a^{\prime}\right)$ for some $a^{\prime} \in C_{q+1}(K)$ then $a$ is called a $q$-boundary. The $q$ th homology group $H_{q}(K)$ of $(K, \partial)$ is the quotient group of $q$-cycles and $q-$ boundaries. The homology class of a chain $a \in C_{q}(K)$ is denoted by $[a]$. If $\mathcal{C}=\left\{C_{q}, \partial_{q}\right\}$ and $\mathcal{C}^{\prime}=\left\{C_{q}^{\prime}, \partial_{q}^{\prime}\right\}$ are chain complexes then a chain map $f: \mathcal{C} \rightarrow \mathcal{C}^{\prime}$ is a family of homomorphisms $\left\{f_{q}: C_{q} \rightarrow \mathcal{C}^{\prime}{ }_{q}\right\}_{q \geq 0}$ such that $\partial_{q}^{\prime} f_{q}=f_{q-1} \partial_{q}$.

It is clear that the problem of computing homology of a cell complex $(K, \partial)$ can be reduced to solving the equation $\partial x=0$ up to boundary. For the actual 
algorithms, we shall follow a computational algebraic approach for computing homology in terms of chain homotopy equivalences, developed first by Eilenberg and Mac Lane in the 1960s 18. This approach was exhaustively used later in algebraic-topological theories like effective homology [19] and homological perturbation theory [20], as well as in discrete settings, like discrete Morse theory [21] and the AT-model 422].

In order to describe our approach to homology computation and to the construction of Alexander-Whitney diagonal, we are going to use the notion of gradient vector fields. Let $(K, \partial)$ be a finite cell complex. A linear map $\phi: C_{*}(K) \rightarrow$ $C_{*+1}(K)$ is called an algebraic gradient vector field (or an algebraic gvf for short) over $K$ if $\phi \phi=0$. An algebraic gvf over $K$ is called a combinatorial gradient vector field (or a combinatorial gvf for short) over $K$ (see 21]) if for any cell $a \in K^{(q)}$, either $\phi(a)=0$, or $\phi(a)=b$ for some $b \in K^{(q+1)}$ (see Fig. 3). An algebraic gvf satisfying the condition $\phi \partial \phi=\phi$ is called an algebraic integral operator. An algebraic integral operator which is only non-null for a unique cell $a \in K^{(q)}$ is called a (combinatorial) integral operator (see [3]). An algebraic integral operator satisfying the additional condition $\partial \phi \partial=\partial$ is called a homology gvf (see [22]).

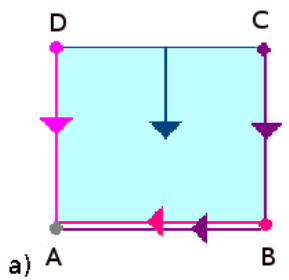

b)

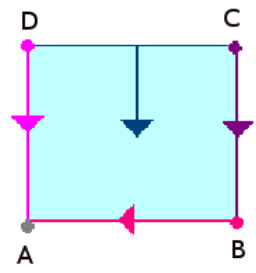

c) $\mathrm{A}$

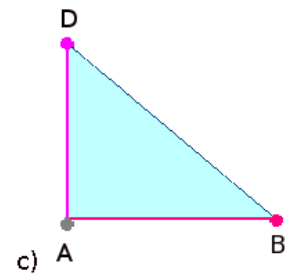

Fig. 3. A sample algebraic gradient vector field (a), a sample combinatorial gradient vector field (b), and the resultant image after applying $\phi(C)=C B(\mathrm{c})$. The difference between (a) and (b) is that $\phi(C)=C B+A B$ in (a), while $\phi(C)=B C$ in (b).

It turns out that a homology gvf over $K$ determines a strong algebraic relationship connecting $C(K)$ and its homology vector space $H(K)$. In order to show this, we use the notion of a chain contraction $(f, g, \phi):(C, \partial) \rightarrow\left(C^{\prime}, \partial^{\prime}\right)$ between two chain complexes, which is a triple of linear maps $f: C_{*} \rightarrow C_{*}^{\prime}, g: C_{*}^{\prime} \rightarrow C_{*}$ and $\phi: C_{*} \rightarrow C_{*+1}$ that satisfy the following conditions: (a) $\operatorname{Id}_{C}-g f=\partial \phi+\phi \partial$; (b) $f g=\operatorname{Id}_{C^{\prime}}$; (c) $f \phi=0$; (d) $\phi g=0$; (e) $\phi \phi=0$. This is a classical notion in homological algebra and algebraic topology, but it is an exotic concept within the digital imagery setting [3234]. The following proposition can be derived in a straightforward manner from [20, using the integral operator language.

Proposition 1. Let $(K, \partial)$ be a finite cell complex. An algebraic integral operator $\phi: C_{*}(K) \rightarrow C_{*+1}(K)$ over $K$ gives rise to a chain contraction $(\pi, \iota, \phi)$ from $C(K)$ onto its chain subcomplex $\operatorname{Im} \pi$, where $\pi$ is a projection and $\iota$ is the inclusion map. Reciprocally, if $(f, g, \phi)$ is a chain contraction from $C(K)$ to another chain complex $C^{\prime}$, then $\phi$ is an algebraic integral operator. 
Note that given an algebraic integral operator, the projection $\pi$ can be instantly determined by the formula $\pi=\operatorname{Id}_{C(K)}-\partial \phi-\phi \partial$.

The following result is a refinement of Proposition 1 and justifies the correctness of the homology computation algorithm that we use.

Proposition 2 (see [7]). Let $(K, \partial)$ be a finite cell complex. A homology gvf $\phi: C_{*}(K) \rightarrow C_{*+1}(K)$ over $K$ gives rise to a chain contraction $(\pi, \iota, \phi)$ from $C(K)$ onto its chain subcomplex isomorphic to the homology of $K$, where $\pi$ is the projection $\pi=\mathrm{Id}_{C(K)}-\partial \phi-\phi \partial$, and $\iota$ is the inclusion map $\iota: H(K) \rightarrow C(K)$. Reciprocally, if $(f, g, \phi)$ is a chain contraction from $C(K)$ to its homology $H(K)$, then $\phi$ is a homology gvf.

We apply the homology computation process given in $[722$, in which the incremental homology algorithm introduced in [1] was adapted for obtaining a homology gvf. We assume that the input cell complex $(K, \partial)$ is given together with filter $\mathcal{K}$ for $K$, that is, an ordered set of cells $\mathcal{K}=\mathcal{K}_{m}=\left\langle c_{1}, \ldots, c_{m}\right\rangle$ such that $\left\{c_{1}, \ldots, c_{m}\right\}=\bigcup_{q \in \mathbb{Z}} K^{(q)}$, and for each $j=1, \ldots, m$, all the faces of $c_{j}$ are contained in the subset $\left\{c_{1}, \ldots, c_{j-1}\right\}$. For each $i=0, \ldots, m$, we represent the cell subcomplex $K_{i}$ of $K$ consisting of $\left\{c_{0}, \ldots, c_{i}\right\}$ by the filter $\mathcal{K}_{i}:=\left\langle c_{0}, \ldots, c_{i}\right\rangle$, and we denote its boundary map by $\partial$. In the algorithm, $\mathcal{H}_{i}$ is a set of generators of a chain complex $H_{i}$ isomorphic with the homology of $K_{i}$. Let us note that $\phi$, $\iota$ and $\pi$ are described in Proposition 2 .

\section{Algorithm 1}

INPUT:

$\mathcal{K}=\left\langle c_{1}, \ldots, c_{m}\right\rangle-$ a filter of a cell complex $(K, \partial) ;$

$\partial$ - the boundary operator on $K$.

Pseudocode:

$\mathcal{H}_{0}:=\left\{c_{0}\right\} ; \phi_{0}\left(c_{0}\right):=0 ; \pi_{0}\left(c_{0}\right):=c_{0} ;$

for $i:=1$ to $m$ do

$\phi_{i}\left(c_{i}\right):=0 ; \pi_{i}\left(c_{i}\right):=\bar{c}_{i}:=c_{i}+\phi_{i-1} \partial\left(c_{i}\right) ;$

if $\partial \bar{c}_{i}=0$ then

for $j:=0$ to $i-1$ do

$\phi_{i}\left(c_{j}\right):=\phi_{i-1}\left(c_{j}\right)$;

$\mathcal{H}_{i}:=\mathcal{H}_{i-1} \cup\left\{\bar{c}_{i}\right\}$

otherwise express $\partial \bar{c}_{i}$ as a sum of elements of $\mathcal{H}_{i-1}$ :

$\partial \bar{c}_{i}=\sum_{j=1}^{r_{i}} u_{j}$, where each $u_{j} \in \mathcal{H}_{i-1}$;

choose any summand $u_{k}$, where $k \in\{1, \ldots, r\}$;

for $j:=0$ to $i-1$ do

$\xi_{j}^{i}:=c_{j}+\left(\phi_{i-1} \partial+\partial \phi_{i-1}\right)\left(c_{j}\right) ;$

express $\xi_{j}^{i}$ as a sum of elements of $\mathcal{H}_{i-1}$ :

$\xi_{j}^{i}=\sum_{l=1}^{t_{i j}} v_{l}$, where each $v_{l} \in \mathcal{H}_{i-1} ;$

if $u_{k}=v_{l}$ for some $l \in\left\{1, \ldots, t_{i j}\right\}$ then

$\phi_{i}\left(c_{j}\right):=\phi_{i-1}\left(c_{j}\right)+\bar{c}_{i}$;

else $\phi_{i}\left(c_{j}\right):=\left(\phi_{i-1}\left(c_{j}\right)\right.$; 


$$
\begin{aligned}
\pi_{i}\left(c_{j}\right):=c_{j}+\left(\phi_{i} \partial+\partial \phi_{i}\right)\left(c_{j}\right) ; \\
\mathcal{H}_{i}:=\mathcal{H}_{i-1} \backslash\left\{u_{k}\right\} .
\end{aligned}
$$

OutPut:

$\mathcal{H}_{m}$ - a set of homology generators of $K$;

$\left(\pi_{m}, \iota, \phi_{m}\right)$ - a chain contraction in which $\phi_{m}$ is a homology gvf over $K$.

The complexity of this algorithm is $O\left(m^{3}\right)$. Note that expressing $\partial \bar{c}_{i}$ and $\xi_{j}^{i}$ as a sum of elements of $\mathcal{H}_{i-1}$ is straightforward, because $\pi$ is compatible with the boundary operator. Therefore, solving the corresponding system of linear equations is trivial, and does not increase the complexity of the algorithm.

The application of the algorithm to a simple cubical set is shown in the Appendix.

\section{Alexander-Whitney Diagonal of a Cubical 2-Cell}

In this section we specify the cubical version of the Alexander-Whithey diagonal for an elementary 2-cube. For that purpose, we use the basic techniques of simplicial cartesian product subdivision and chain homotopy equivalence.

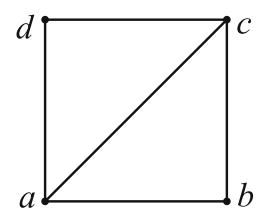

Fig. 4. Simplicial complex of a 2-cube (square): two 2-simplices (triangles), five 1simplices (edges), and four 0-simplices (vertices)

Let $Q$ be the cubical complex of an elementary $2-$ cube and let $S$ be one simplicial subdivision, as illustrated in Fig.4. Consider the combinatorial integral operator $\phi: C_{*}(S) \rightarrow C_{*+1}(S)$ such that $\phi(a c)=a c d$. By Proposition 1 $\phi$ gives rise to a chain contraction $(\pi, \iota, \phi)$ from $C(S)$ to its chain subcomplex $\operatorname{Im}(\pi)$. In this example $\pi(a c)=a b+c b, \pi(a b c)=0, \pi(a c d)=a c d+a b c$ and $\pi(\sigma)=\sigma$ for the rest of elements.

We now use the chain contraction $(\pi, \iota, \phi)$ to transfer the simplicial AlexanderWhitney diagonal into the cubical setting (from $C_{*}(S)$ to $C_{*}(Q)$ ). Recall from 17. that if $\left(w_{1}, w_{2}, w_{3}\right)$ is a 2 -simplex (a triangle) then

$$
\begin{aligned}
A W\left(\left(w_{1}, w_{2}, w_{3}\right)\right)= & \left(w_{1}\right) \otimes\left(w_{1}, w_{2}, w_{3}\right)+ \\
& +\left(w_{1}, w_{2}\right) \otimes\left(w_{2}, w_{3}\right)+\left(w_{1}, w_{2}, w_{3}\right) \otimes\left(w_{3}\right)
\end{aligned}
$$

where $A W: C_{*}(S) \rightarrow C_{*}(S) \otimes C_{*}(S)$.

From the homological point of view, the only nontrivial term in this decomposition is the second one, so one can consider the Alexander-Whitney diagonal to decompose the 2-simplex $\left(w_{1}, w_{2}, w_{3}\right)$ into the product of two 1-simplices $\left(w_{1}, w_{2}\right) \otimes\left(w_{2}, w_{3}\right)$, as illustrated in Fig. 5 . 

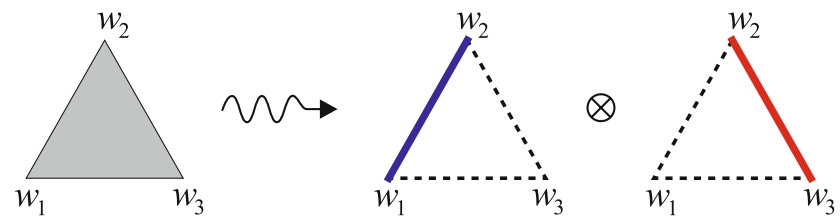

Fig. 5. Decomposition of a 2-simplex (a triangle) into 1-simplices (segments) via the Alexander-Whitney diagonal

A formula for the cubical Alexander-Whitney diagonal on the 2-cube abcd (see Fig. (4) transferred from the simplicial version of the Alexander-Whitney diagonal by means of the formula $A W_{c}(a b c d)=\pi \otimes \pi(A W(\iota(a b c d)))$ is as follows:

$$
A W_{c}(a b c d)=b \otimes a b c d+a b c d \otimes a+b c \otimes c d+b c \otimes a d+c d \otimes a d+a d \otimes a d .
$$

where $A W_{c}: C_{*}(Q) \rightarrow C_{*}(Q) \otimes C_{*}(Q)$.

Note that $\iota(a b c d)=a b c+a c d$. In particular, the result is a combination in which the first two summands are trivial (as the product of the 2-cube and a vertex), and the next four summands are products of elementary cubes of dimension 1 (see Fig. 6).

We shall show in the next section how to extend this formula from a single $2-$ cube to entire 2 -chains that enclose cavities in a cubical object.

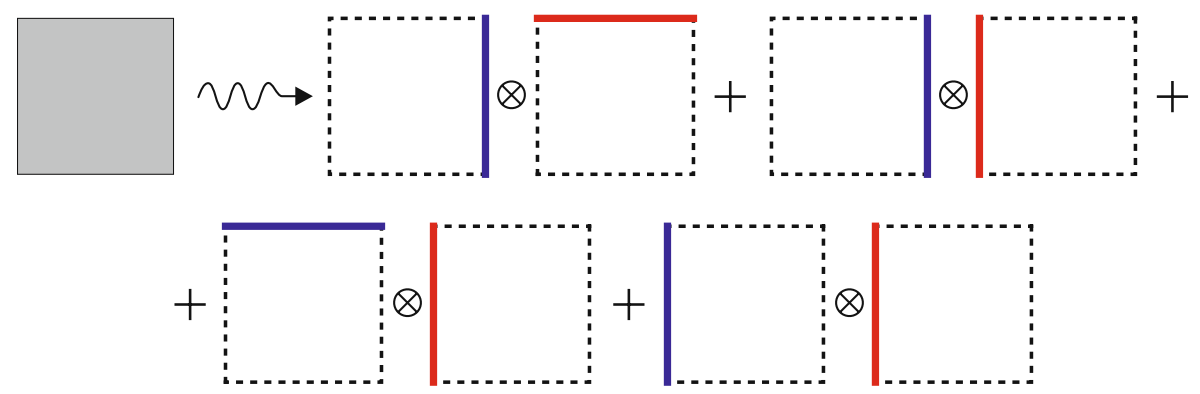

Fig. 6. Decomposition of a cubical 2-cell into a product of 1-cells via the cubical version of the Alexander-Whitney diagonal

\section{Computing the Alexander-Whitney Diagonal for Cavities in a Digital Volume}

In this section, we combine the results of the previous sections into an algorithmic method for determining 2-cycles (cavities) in a digital volume and decomposing them into products of $1-$ cycles. 
Let $(K, \partial)$ be a cubical complex of a binary digital volume, as described in Section 2, Let $\mathcal{K}$ be a filter for $K$. A straightforward filter for $K$ can be constructed by first considering all the 0 -cells in a certain order, then all the 1 -cells, and so on.

Let $\mathcal{H}=\left\{\bar{c}_{1}, \ldots, \bar{c}_{r}\right\}$ and $(\pi, \iota, \phi)$ be the output of Algorithm 1 applied to $\mathcal{K}$ and $\partial$. Let $\left\{\bar{c}_{s_{1}}, \ldots, \bar{c}_{s_{t}}\right\}$ be the 2 -dimensional elements of $\mathcal{H}$. If this set is empty then there are no cavities to decompose. Otherwise, the set $\left\{\bar{c}_{s_{1}}, \ldots, \bar{c}_{s_{t}}\right\}=$ $\mathcal{H} \cap C_{2}(K)$, where $t \in\{1, \ldots, r\}$, consists of representative 2 -cycles in $K$ that correspond to the generators of $H_{2}(K)$. In particular, each $\bar{c}_{s_{i}}=\sum_{j=1}^{t_{i}} c_{i j}$ is a sum of elementary $2-$ cubes enclosing at least one cavity in $K$.

We apply the formula (1) derived in Section 4 for the Alexander-Whitney diagonal to each $c_{i j}, i=1, \ldots, t, j=1, \ldots, t_{i}$, and we obtain a sum of tensor products $A W_{c}\left(\bar{c}_{s_{i}}\right)=\sum_{j=1}^{r_{i}} a_{i j} \otimes b_{i j}$, where each $a_{i j}$ and $b_{i j}$ is a 1 -cube. Finally, we apply the map $\pi$ to these pairs of chains, and in this way we obtain a description of the Alexander-Whitney diagonal in cubical homology: $A W_{c}: H_{2}(K) \rightarrow H_{1}(K) \otimes H_{1}(K)$.

\section{Examples and Conclusions}

In this paper, we introduced an algorithmic method for decomposing cavities in digital volumes into products of cycles. In this section, we show some specific examples that illustrate the usefulness of the Alexander-Whitney diagonal for the analysis of binary digital volumes.

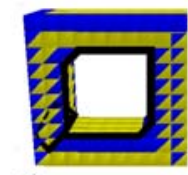

a)

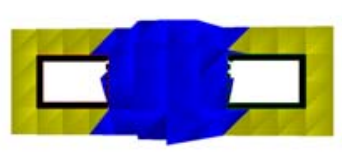

b)
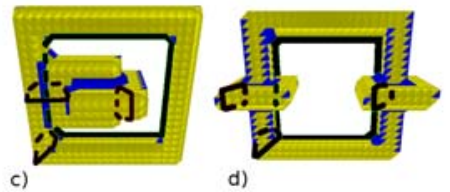

d)

Fig. 7. Sample digital volumes with cavities: (a) a topological torus, (b) a topological sphere with two handles, (c) a configuration with three tori, (d) another configuration with three tori. 1-cycles are indicated in each example.

Let us consider the examples illustrated in Fig. 7. As shown in Table 1, the digital volumes (a) and (b) have the same homological information in terms of Betti numbers (number of connected components $\left(\beta_{0}\right)$, number of 1 -cycles $\left(\beta_{1}\right)$ and number of cavities $\left.\left(\beta_{2}\right)\right)$. But computing the Alexander-Whitney diagonal, however, we can easily distinguish them.

The null result of the Alexander-Whitney diagonal in (b) is due to the fact that the cavity described by the sphere does not present non-trivial 1-cycles.

Similar situation occurs with examples (c) and (d). They have identic Betti numbers, but in order to distinghish them, we have to solve the problem of 
distinguishing the decompositions in terms of homology generators. This problem can be reduced to the problem of an isomorphism of groups in algebra, which is a problem of high computational complexity in general. We do not focus on this problem in this paper, which will be our aim in future work. Let us restrict to say that we can differenciate the respecting decomposition in (c) and (d) using matrix arguments.

\section{References}

1. Delfinado, C., Edelsbrunner, H.: An incremental algorithm for Betti numbers of simplicial complexes on the 3-sphere. Comput. Aided Geom. Design 12, 771-784 (1995)

2. Dey, T., Guha, S.: Computing homology groups of simplicial complexes in $\mathbb{R}^{3}$. Journal of the ACM 45(2), 266-287 (1998)

3. González-Diaz, R., Jiménez, M., Medrano, B., Molina-Abril, H., Real, P.: Integral operators for computing homology generators at any dimension. In: RuizShulcloper, J., Kropatsch, W.G. (eds.) CIARP 2008. LNCS, vol. 5197, pp. 356-363. Springer, Heidelberg (2008)

4. González-Diaz, R., Real, P.: On the cohomology of $3 d$ digital images. Discrete Applied Math. 147, 245-263 (2005)

5. Kaczynski, T., Mischaikow, K., Mrozek, M.: Computational homology. Applied Mathematical Sciences (2004)

6. Mischaikow, K., Mrozek, M., Pilarczyk, P.: Graph approach to the computation of the homology of continuous maps. Foundations of Computational Mathematics 5(2), 199-229 (2005)

7. Molina-Abril, H., Real, P.: Cell at-models for digital volumes. In: Torsello, A., Escolano, F., Brun, L. (eds.) GbRPR 2009. LNCS, vol. 5534, pp. 314-323. Springer, Heidelberg (2009)

8. Mrozek, M., Pilarczyk, P., Żelazna, N.: Homology algorithm based on acyclic subspace. Computers and Mathematics with Applications 55, 2395-2412 (2008)

9. Peltier, S., Alayrangues, S., Fuchs, L., Lachaud, J.: Computation of homology groups and generators. Computer \& Graphics 30(1), 62-69 (2006)

10. Computational Homology Project, http://chomp.rutgers.edu/

11. Gameiro, M., Mischaikow, K., Kalies, W.: Topological Characterization of SpatialTemporal Chaos. Physical Review E 703 (2004)

12. Gameiro, M., Pilarczyk, P.: Automatic homology computation with application to pattern classification. RIMS Kokyuroku Bessatsu B3, 1-10 (2007)

13. Krishan, K., Gameiro, M., Mischaikow, K., Schatz, M., Kurtuldu, H., Madruga, S.: Homology and symmetry breaking in Rayleigh-Bénard convection: Experiments and simulations. Physics of Fluids 19, 117105 (2007)

14. Niethammer, M., Stein, A., Kalies, W., Pilarczyk, P., Mischaikow, K., Tannenbaum, A.: Analysis of blood vessel topology by cubical homology. In: Proc. of the International Conference on Image Processing, vol. 2, pp. 969-972 (2002)

15. Żelawski, M.: Pattern recognition based on homology theory. Machine Graphics and Vision 14, 309-324 (2005)

16. Serre, J.: Homologie singulière des espaces fibrés, applications. Annals of Math. 54, 429-505 (1951) 
17. Hatcher, A.: Algebraic Topology. Cambridge University Press, Cambridge (2001)

18. Eilenberg, S., Mac Lane, S.: On the groups $h(\pi, n)$, i, ii, iii. Annals of Math. 58, 60, 60, 55-106,48-139, 513-557 (1953, 1954)

19. Sergeraert, F.: The computability problem in algebraic topology. Advances in Mathematics 104, 1-29 (1994)

20. Barnes, D.W., Lambe, L.A.: A fixed point approach to homological perturbation theory. Proc. Amer. Math. Soc. 112, 881-892 (1991)

21. Forman, R.: A Discrete Morse Theory for Cell Complexes. In: Yau, S.T. (ed.) Topology and Physics for Raoul Bott. International Press (1995)

22. Molina-Abril, H., Real, P.: Advanced homological information on 3d digital volumes. In: da Vitoria Lobo, N., Kasparis, T., Roli, F., Kwok, J.T., Georgiopoulos, M., Anagnostopoulos, G.C., Loog, M. (eds.) S+SSPR 2008. LNCS, vol. 5342, pp. 361-371. Springer, Heidelberg (2008)

23. González-Diaz, R., Medrano, B., Real, P., Sanchez-Pelaez, J.: Algebraic topological analysis of time-sequence of digital images. In: Ganzha, V.G., Mayr, E.W., Vorozhtsov, E.V. (eds.) CASC 2005. LNCS, vol. 3718, pp. 208-219. Springer, Heidelberg (2005)

\section{Appendix. Example for Algorithm 1}

Sample application of the homology computation algorithm to a simple cell complex illustrated in Fig. 8 has been shown in Table 2

Table 1. Betti numbers and the Alexander-Whitney diagonal computed for the examples shown in Fig. 7. Generators of $H_{1}$ are labeled $\alpha_{i}$, generators of $H_{2}-\gamma_{j}$.

\begin{tabular}{|c|c|c|c|c|}
\hline Example & $\beta_{0}$ & $\beta_{1}$ & $\beta_{2}$ & $A W_{c}$ \\
\hline$(a)$ & 1 & 2 & 1 & $\gamma_{1}=\alpha_{1} \otimes \alpha_{2}$ \\
$(b)$ & 1 & 2 & 1 & 0 \\
$(c)$ & 1 & 4 & 3 & $\gamma_{1}=\alpha_{1} \otimes \alpha_{3}, \gamma_{2}=\alpha_{2} \otimes \alpha_{3}, \gamma_{3}=\alpha_{4} \otimes \alpha_{3}$ \\
$(d)$ & 1 & 4 & 3 & $\gamma_{1}=\alpha_{1} \otimes \alpha_{2}, \gamma_{2}=\alpha_{2} \otimes \alpha_{3}, \gamma_{3}=\alpha_{2} \otimes \alpha_{4}$ \\
\hline
\end{tabular}
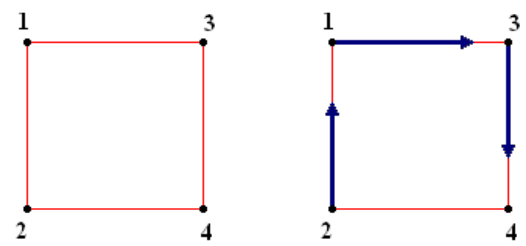

Fig. 8. A sample cell complex and a homology gvf for it expressed in a pictorial way 
Table 2. Monitoring of algorithm 1] using the complex shown in Fig. 8

\begin{tabular}{|c|c|c|c|c|c|c|c|c|}
\hline \begin{tabular}{|l|}
$i$ \\
\end{tabular} & 0 & 1 & 2 & 3 & 4 & 5 & 6 & 7 \\
\hline$\sigma$ & $\langle 1\rangle$ & $\langle 2\rangle$ & $\langle 3\rangle$ & \begin{tabular}{|l|}
$\langle 4\rangle$ \\
\end{tabular} & $\langle\langle 1,2\rangle$ & $\langle 1,3\rangle$ & $\langle 2,4\rangle$ & $\langle 3,4\rangle$ \\
\hline $\begin{array}{l}\pi \\
\phi\end{array}$ & $\begin{array}{c}1\rangle \\
0\end{array}$ & & & & & & & \\
\hline $\begin{array}{l}\pi \\
\phi\end{array}$ & $\begin{array}{c}\langle 1\rangle \\
0\end{array}$ & $\begin{array}{c}\langle 2\rangle \\
0\end{array}$ & & & & & & \\
\hline $\begin{array}{l}\pi \\
\phi\end{array}$ & $\begin{array}{c}\langle 1\rangle \\
0\end{array}$ & $\begin{array}{l}\langle 2\rangle \\
0\end{array}$ & $\begin{array}{c}\langle 3\rangle \\
0\end{array}$ & & & & & \\
\hline $\begin{array}{l}\pi \\
\phi \\
\end{array}$ & $\begin{array}{c}1\rangle \\
0\end{array}$ & $\begin{array}{c}2\rangle \\
0\end{array}$ & $\begin{array}{c}3\rangle \\
0\end{array}$ & \begin{tabular}{|c|}
$44\rangle$ \\
0
\end{tabular} & & & & \\
\hline \begin{tabular}{|l|l|}
$\pi$ \\
$\phi$
\end{tabular} & $\begin{array}{c}\langle 2\rangle \\
\langle 1,2\rangle\end{array}$ & $\begin{array}{c}\langle 2\rangle \\
\langle 0\end{array}$ & $\begin{array}{c}\langle 3\rangle \\
0\end{array}$ & \begin{tabular}{|c|}
$\langle 4\rangle$ \\
0
\end{tabular} & $\begin{array}{c}\langle 1,2\rangle \\
0\end{array}$ & & & \\
\hline $\begin{array}{ll}\pi \\
0\end{array}$ & $\begin{array}{l}\langle 3\rangle \\
\langle 1,3\rangle\end{array}$ & $\begin{array}{c}\langle 3\rangle \\
\langle 1,2\rangle+\langle 1,3\rangle\end{array}$ & $\begin{array}{c}3\rangle \\
0\end{array}$ & \begin{tabular}{|c|}
$\langle 4\rangle$ \\
0
\end{tabular} & $\begin{array}{l}0 \\
0\end{array}$ & $\langle\underset{0}{\langle 1,3\rangle+\underset{ }{+}\langle 1,2\rangle}$ & & \\
\hline $\begin{array}{l}\pi \\
\phi\end{array}$ & $\langle 1,2\rangle+\langle 2,4\rangle$ & $\begin{array}{c}\langle 4\rangle \\
\langle 2,4\rangle\end{array}$ & $\langle 2,4\rangle+\langle 1,2\rangle+\langle 1,3\rangle$ & \begin{tabular}{|c|}
$\langle 4\rangle$ \\
0
\end{tabular} & $\begin{array}{l}0 \\
0\end{array}$ & $\begin{array}{l}0 \\
0\end{array}$ & $\langle 2,4\rangle+\underset{0}{\langle 1,2\rangle}+\langle 1,3\rangle$ & \\
\hline $\begin{array}{l}\pi \\
\end{array}$ & $\begin{array}{c}\langle 4\rangle \\
\langle 1,2\rangle+\langle 2,4\rangle\end{array}$ & $\begin{array}{c}\langle 4\rangle \\
\langle 2,4\rangle\end{array}$ & $\begin{array}{c}\langle 4\rangle \\
\langle 2,4\rangle+\langle 1,2\rangle+\langle 1,3\rangle\end{array}$ & \begin{tabular}{|c|}
$\langle 4\rangle$ \\
0
\end{tabular} & 0 & $\begin{array}{l}0 \\
0\end{array}$ & $\begin{array}{c}\langle 2,4\rangle+\langle 1,2\rangle+\langle 1,3\rangle \\
0\end{array}$ & $\begin{array}{c}\langle 3,4\rangle+\langle 2,4\rangle \\
+\langle 1,2\rangle+\langle 1,3\rangle \\
0\end{array}$ \\
\hline
\end{tabular}

The intermediary homology results are

$H_{1}=\langle 1\rangle ; H_{2}=\langle 1\rangle,\langle 2\rangle ; H_{3}=\langle 1\rangle,\langle 2\rangle,\langle 3\rangle ; H_{4}=\langle 1\rangle,\langle 2\rangle,\langle 3\rangle,\langle 4\rangle ; H_{5}=\langle 2\rangle,\langle 3\rangle,\langle 4\rangle ; H_{6}=\langle 3\rangle,\langle 4\rangle ; H_{7}=\langle 4\rangle ; H_{8}$ $=\langle 4\rangle,\langle 3,4\rangle+\langle 2,4\rangle+\langle 1,2\rangle+\langle 1,3\rangle$ 